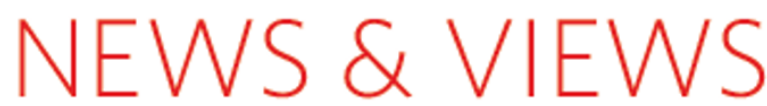

IMMUNOLOGY

\title{
Protection and privilege
}

\author{
Herman Waldmann
}

\section{The immune system not only attacks microbes, but also regulates itself to avoid harming vital organs. Cells notorious for their involvement in allergy turn out to be vital to this protective function.}

The immune system's capacity to regulate its own activities can be exploited to prevent rejection of transplanted organs and to reverse autoimmune diseases such as diabetes. The chief agents of this immune self-regulation system are specialized cells $-T$ regulatory cells $\left(\mathrm{T}_{\mathrm{reg}}\right)$ - that somehow prevent other immune cells from attacking healthy tissues. In this issue (page 997) ${ }^{1}$, Lu et al. demonstrate that the protective actions of $\mathrm{T}_{\text {reg }}$ cells depend on so-called mast cells, which were hitherto bad-mouthed for their undesirable role in allergic responses*. Perhaps, at last, a virtuous physiological role for mast cells has been uncovered.

Long-term tolerance of transplanted tissues by the body can be achieved by using a short treatment of antibodies that block vital co-receptors (CD4 and CD8) or co-stimulatory molecules (CD154) on a subset of immune cells ( $\mathrm{T}$ cells). This reprogramming of the immune system depends on the activity of specialized regulatory $\mathrm{CD}^{+} \mathrm{T}$ cells $\left(\mathrm{T}_{\text {reg }}\right)$ that are drawn to the transplanted tissue and its surrounding milieu. The $\mathrm{T}_{\text {rog }}$ cells confer on the tissue some special exemption from attack, just as the fetus in the womb is protected from the mother's immune system. A major goal of current immunological research is to establish just how $\mathrm{T}_{\text {rg }}$ cells ensure this ceasefire.

A clue to what that mechanism is came from the observation that cultures of $\mathrm{T}_{\text {reg }}$ cells tend to be contaminated with 'unwanted mast cells'. This was attributed to a secreted molecule called IL-9 that is made by the $T_{\text {reg }}$ cells and that enhances mast cell growth and functionality. More surprisingly, when recipient mice that were tolerating a skin graft were given a second graft from an identical donor, the second graft became infiltrated not only with $\mathrm{T}_{\mathrm{reg}}$ cells, but also with mast cells ${ }^{3}$. This led to the proposal that $\mathrm{T}_{\mathrm{reg}}$ and mast cells might form a functional unit that mediates graft tolerance $\mathrm{c}^{2,3}$.

Lu et al. ${ }^{1}$ confirm these findings and extend them by performing crucial work using an experimental mouse strain (C57BL/6-

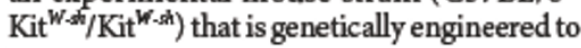

'This article and the paper concerned'were published online on 20 August 2006.

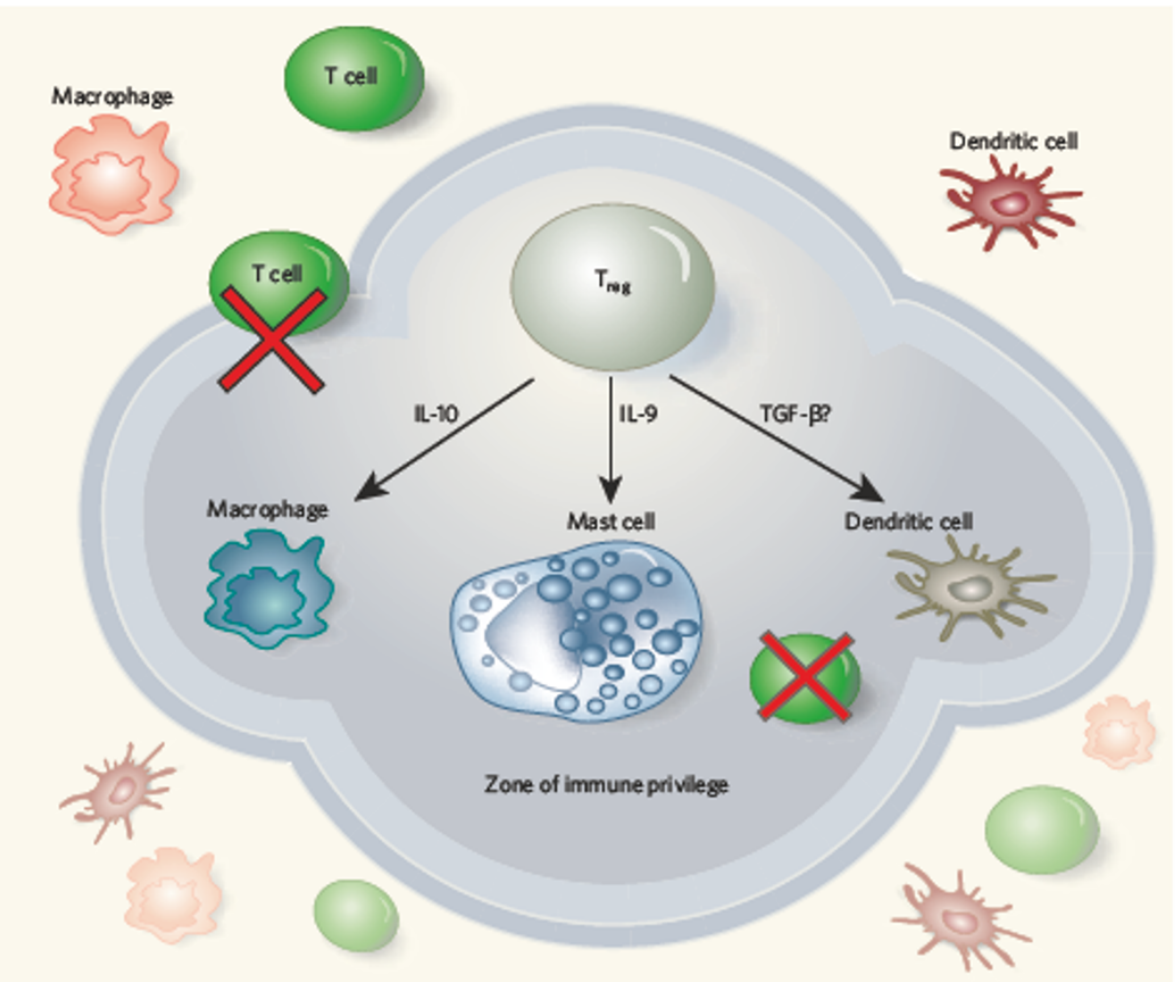

Figure 1 | Immune self-regulation: a poss ible mechanism. The work of Lu et al. ${ }^{1}$ suggests that mast cells interact with regulatory $\mathrm{T}$ cells $\left(\mathrm{T}_{\mathrm{reg}}\right)$ to exempt transplanted tissues from an immune response. If sufficient $T_{\mathrm{ng}}$ cells can enter a transplanted tissue, they could then mobilize mast cells to enhance the capacity of the mast cells to decommission immune responses within a limited 'privileged' area. By interacting with antigen in the tissue, the $T_{n g}$ cells are activated to produce the IL-9 polypeptide and perhaps other molecules (maybe IL-10 or TGF- $\beta$ ). These molecules enable $\mathrm{T}_{\text {reg }}$ cells to interact with cells of the innate immune system (such as dendritic cells or macrophages), leading to further inhibition of immune responses. The end result is that $T$ cells, which might otherwise kill other cells and damage tissues, are prevented from doing so and are then inactivated.

be deficient in mast cells". The authors used these mice as recipients for transplants, but their attempts to induce therapeutic tolerance in the mice were unsuccessful, as all the test grafts were promptly rejected. Spectacularly, however, injecting mast-cell-enriched cell cultures into the recipient mice restored the host's capacity to accept grafts for prolonged periods.

The second major breakthrough by Lu et al. ${ }^{1}$ comes from the discoveries that all categories of $\mathrm{T}_{\text {reg }}$ cell make IL-9, that IL-9 can be found in the tolerated graft, and that neutralization of IL-9 in vivo prevents regulation of graft rejection by $\mathrm{T}_{\text {reg }}$ cells. The authors interpret the findings as indicating that IL-9 from $\mathrm{T}_{\text {reg }}$ cells is involved in the recruitment and activation of mast cells at the graft site.

Mast cells belong to the 'innate' immune system - the system that provides immediate defence against microbes that the body hasn't encountered before. Although they participate in immune responses, they do not intrinsically recognize unique antigens. The recognition of 
specific antigens is mediated instead by the 'adaptive' immune system, where cells such as $T$ cells remember previous microbe encounters and react strongly against the aliens if the infection is repeated.

When it comes to immunity towards microbes, cells of the innate immune system, including mast cells, macrophages and dendritic cells, interact with the adaptive system in two ways. First, they sense dangerous microbes through generic receptors for potential pathogens, and, once activated, they alert the adaptive system to the danger. Mast cells, for instance, expel packets of mediator molecules (including histamine) that cause an immediate inflammatory reaction and attract adaptive cells. Second, innate immune cells can act as the executive arm of the adaptive system, once armed with antibodies or mobilized to killer-mode by T-cell-derived mediators.

Recent research on macrophages and dendritic cells has taught us that the innate immune system may also mediate the opposing process of permanently decommissioning adaptive cells so that they cannot mount an immune response. For example, immature dendritic cells display antigens in a way that makes $\mathrm{T}$ cells tolerate that antigen so they no longer respond to it. Moreover, $\mathrm{T}_{\text {reg }}$ cells can manifest their suppressive activity by modulating dendritic cells, so that the dendritic cells are no longer able to alert the adaptive cells to danger.

Perhaps thissymmetry - in both activating and inhibiting adaptive reactions - is a general feature of innate cells. We have tended to think of these cells as agents of destruction, waiting around until the microbe appears. Now, there are reasonable grounds for elevating their status to cells that have a positive physiologi$\mathrm{cal}$ role - ensuring self-tolerance and/or tissue integrity through the maintenance of 'privileged' microenvironments where adaptive immune responses are damped down (Fig. 1).

The implications of the Lu et $a L^{1}$ paper go beyond transplantation. This work could form the basis for understanding why mast cells are located in very specific sites within tissues (for example, nerves, vessels, hair follicles or epithelia). It was always hard to see how this positioning was related to their potential for immune function. Perhaps these sites require some low-level immune privilege. Also, is it possible that the mast cells found within tumours contribute some immune privilege? In support of this idea, mast-cell-deficient mice seem to have some natural resistance to the induction of tumours ${ }^{5}$.

Which checkpoint of the immune selfregulatory response needs the mast cells is not yet established. Are they required to induce $\mathrm{T}$ cells to a regulatory role or are they required at some later phase in the execution of that function? Either way, the current findings will galvanize research into the molecular basis by which mast cells decommission immune function, and provide a possible new direction for acquiring drug targets.

Herman Waldmann is in the Sir William Dunn School of Pathology, University of Oxford, South Parks Road, Oxford OX13RE, UK. e-mail: herman.waldmann@path.ox.ac.uk
1 Lu, L-F. et al. Nature 442, 997-1002(2006)

2. Cobbold, S. P.et al. Immunal. Rex 196, 109-124 (2003).

3. Zelenika, D.et al. Immunol. Rex 182, 164-179 (2001).

4. Galli, S.I, Nakae, S.\& Tsai, M. Nature Immunal. 6, 135-142 (2005).

5. Wedemeyer, L\& Galli, S. I. Lab. Invest. 85, 388-396 (2005).

\section{COSMOLOGY}

\section{Unique, or not unique?}

\section{Martin Bojowald}

\section{That is the question. The search for a single theory of everything is as old as science itself, and is now the beat of quantum cosmologists. But some basic tenets that inform the quest are being challenged.}

Whether 'tis nobler in the mind to suffer The slings and arrows of outrageous fortune, Or to take arms against a sea of troubles, And, by opposing, end them?

Hamlet Act III, Scene i

Hamlet's existential agony has always been, in a variant form, part of the mindset of those researching quantum gravity. In this field, a large faction has deemed itself to be in the outrageously fortunate position of being close to a unique theory of how the Universe is as it is. This theory would not only put descriptions of all forms of matter and their interactions on the same footing, but also reconcile the two seemingly inharmonious pillars of modern physics: general relativity, which describes gravity, and quantum theory. The belief in such an all-encompassing theory has been the driving force for the various models known as string theories, grouped under the umbrella of 'M-theory', that have been developed in the past decades.

But there is an alternative concept. This holds that solutions, rather than models, are unique. Solutions are generally more important for physics than theories: observations are compared with properties that emerge from a theory, not with the construct itself. But finding realistic solutions to theories - solutions that reproduce the features of the Universe that we observe now - has proved much harder and messier than constructing the theories themselves.

That applies even with a modest interpretation of 'realistic', such as merely requiring the solution to undergo the kind of accelerated late expansion that our Universe appears to be going through now. Once such solutions were finally uncovered ${ }^{1}$, myriads of them turned up in various corners of the string landscape ${ }^{2}$. Thus, as there is currently no selection criterion
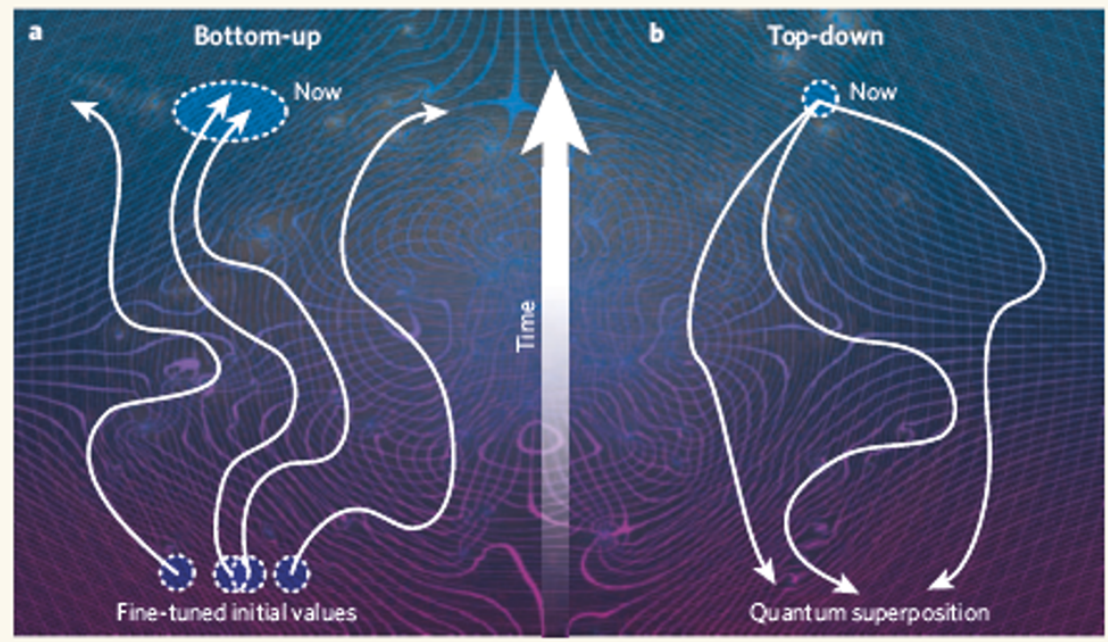

Figure 1| Take it from the top. a, A bottom-up description requires fine-tuned initial values for individual histories to arrive close to a universe as we see it now. b, A top-down formulation avoids that problem by starting with present properties and working backwards. Both viewpoints can be used in classical and quantum physical frameworks, but the top-down interpretation is most strongly motivated by quantum cosmology. The quantum top-down approach picks suitable histories from a quantum superposition of all possible histories that lead to the current Universe. Thus here, the solution, rather than the theory that leads to it, is unique. 